\title{
(t) \\ DISPUTA ENTRE TEORÍA Y PRÁCTICA EN EL TRABAJO SOCIAL MEXICANO
}

\author{
Dispute between theory and practice in mexican social work
}

\section{Berenice Pérez Ramirez}

\section{RESUMO}

Na Escuela Nacional de Trabajo Social (ENTS) da Universidad Nacional Autónoma de México (UNAM) há um eixo muito importante chamado práticas escolares integrado por: prática comunitária, prática regionalizada e prática da especialização. Para cada uma das práticas mencionadas se realiza um acompanhamento aos alunos durante um ano, que correspondem a dois semestres consecutivos, as atividades desenvolvidas são: realização de diagnóstico social; seguida da intervenção social. Devido a essa experiência, neste ensaio me interessa discutir a articulação entre a teoria e a pratica social. A reflexão busca demonstrar que apesar da afirmação da estreita vinculação entre teoria e prática, esses campos são visualizados de maneira separada o que acarreta uma série de contradições e problemas na nossa profissão, pois não se percebe relevante que as contradições apresentadas sejam debatidas no processo de formação acadêmica. Portanto, a reflexão transita no que foi encontrado nas aulas, a partir dos interesses, das demandas e das angústias dos estudantes, refletindo em variadas interpretações sobre o Serviço Social, no contexto atual. Busca-se na primeira parte do ensaio, revisar conceitos que formam parte do eixo do discurso hegemônico para o Serviço Social no México. Na segunda parte, irei analisar o campo de contradições que permeiam nossa formação acadêmica, bem como, reconhecer a relevância daa categoria de análise: contradição. Finalmente, na terceira parte, pretendo colocar em discussão o sentido da práxis como possibilidade norteadora da intervenção

\footnotetext{
${ }^{1}$ Licenciada en Trabajo Social. Doctorado en Sociología. Profesora-Investigadora de Tiempo Completo en la Escuela Nacional de Trabajo Social (UNAM, Ciudad de México, México). Avenida Universidad 3000, Cd. Universitaria, 04350 Ciudad de México, CDMX, México. E-mail: <bereberes8791@gmail.com>.
} 
para o Serviço Social na realidade mexicana, considerando os aspectos: político, econômico y social desta sociedade.

\section{PALAVRAS-CHAVE}

Serviço social. Teoría e prática. Intervenção. Capitalismo.

\section{ABSTRACT}

At Escuela Nacional de Trabajo Social (ENTS) of Universidad Nacional Autónoma de México(UNAM) there is a very important axis called school practices integrated by: community practice, regional practice and practice of specialization. For each one, students are followed up for a year, which correspond to two consecutive semesters. The activities developed are: social diagnosis; followed by social intervention. Due to this experience, in this essay I am interested in discussing the articulation between theory and social practice. The reflection seeks to demonstrate that despite the affirmation of the close link between theory and practice, these fields are viewed in a separate way, which involve a series of contradictions and problems in our profession, because the contradictions are not relevant in the discussing into the process of academic education. Therefore, the reflection is linked with I have found in the classes, from the interests, the demands and the anguish of the students, reflecting in varied interpretations on the Social Service, in the current context. In this essay, to review concepts that are part of the axis of the hegemonic discourse for Social Service in Mexico. In the second part, I will analyze the field of contradictions that cross our academic formation, as well as recognize the relevance of contradiction. Finally, in the third part, I intend to discuss the meaning of praxis as a guiding possibility of intervention for Social Service in the Mexican reality, considering the political, economic and social aspects of this society.

\section{KEYWORDS}

Social work. Theory and practice. Intervention. Capitalism.

Submetido em: 15/5/2017

Aceito em: 7/8/2017

\section{INTRODUCCIÓN}

Desde enero de 2014 me incorporé como docente a la Escuela Nacional de Trabajo Social (ENTS) de la Universidad Nacional Autónoma de México (UNAM), teniendo a mi cargo un grupo de práctica comunitaria, una asignatura que dura un año escolar ${ }^{2}$. La formación en la licenciatura de trabajo social en la UNAM, tiene como eje principal las prácticas escolares: práctica comunitaria ( $4^{\circ}$ y $5^{\circ}$ semestre), práctica regional $\left(6^{\circ}\right.$ y $7^{\circ}$ semestre) y práctica de especialización ( $8^{\circ}$ y $9^{\circ}$ semestre). En las tres modalidades,

\footnotetext{
2 Actualmente continúo trabajando en esta entidad académica pero ahora imparto práctica de especialización, una clase en el Programa de Maestría en Trabajo Social y coordino un proyecto de investigación.
} 
se establece que en la primera mitad del año, se deberá realizar la elaboración de un diagnóstico social y, para la segunda mitad, se estima que se desarrollará la intervención social. Es decir, en el primer semestre será la investigación y durante el segundo la intervención. El estudiantado describe su formación de la siguiente manera: antes del $4^{\circ}$ semestre ve la teoría y luego la práctica, durante las prácticas escolares. Por el título de las asignaturas de práctica, el estudiantado espera estar en campo la mayor parte del tiempo y desarrollando actividades con las personas de la comunidad o las instituciones.

En el año 2014 durante la práctica comunitaria, me propuse revisar lecturas sobre intervenciones desde distintos enfoques: psicológico, psicoanalítico, educativo, artístico, y por supuesto, desde trabajo social. Una de las primeras interrogantes que hizo el alumnado era ¿es la intervención el acto distintivo de trabajo social? Yo respondí, averigüémoslo leyendo las intervenciones que se hacen desde otras disciplinas. Una segunda interrogante era ¿no estamos destinando tiempo a la lectura que debería ser ocupado en la intervención, en la práctica? Fue esta última pregunta la que detonó, otra serie de cuestionamientos. Con base en esta experiencia, la cuestión de la intervención social se ha vuelto un asunto interesante para pensar sobre nuestra profesión en el contexto mexicano. Algunas de las preguntas que surgieron son: ¿Por qué la urgencia de "intervenir"? ¿La lectura, el análisis y la escritura, no son intervenciones? ¿El estudiantado pensará que una vez concluido el diagnóstico es evidente en qué y con quiénes hay que intervenir? y finalmente ¿intervenir para qué?

El objetivo en este ensayo es debatir en torno a la articulación de la teoría y la práctica. Aun cuando reivindicamos su estrecha vinculación, en el contexto mexicano les vemos como campos separados y eso conlleva una serie de contradicciones y problemas en trabajo social. Contrapuntearemos esta reflexión con lo encontrado en el aula; los intereses, demandas y angustia del alumnado han funcionado como brújula para hacer un acercamiento sobre la siguiente cuestión ¿qué estamos entendiendo por trabajo social? Una hipótesis es que en ocasiones se le imagina más como un espacio de formación en activismo que como un espacio de formación académica. 
El objetivo más amplio es presentar algunos elementos del discurso disciplinar dominante de trabajo social en México, que no es el único discurso, pero sí el más presente. También me interesa apuntar la interrelación que tiene con el contexto global. Es necesario cuestionarnos acerca de lo que hacemos, tanto en la formación como en el campo profesional, reiterando nuevamente que es un continuo, y no dos espacios disociados. Reflexionar sobre las prácticas, saberes, discursos, imaginarios y experiencias en torno a una disciplina, debe ser una tarea permanente que, además, nos lleve a superar viejas dicotomías y analizar problemas cada vez más complejos. Por lo pronto, platearemos algunos elementos que nos permitirán ahondar en esta discusión -que dicho sea de paso, no es reciente - incluso vivida como disputa, entre la teoría y la práctica.

En la primera parte expondremos algunos conceptos que son eje del discurso hegemónico en trabajo social en México. En la segunda parte, desarrollaremos el término contradicción y explicaremos por qué es relevante atender a estas contradicciones en nuestra formación. Finalmente en la tercera parte, exponemos los posibles horizontes de trabajo social en nuestro contexto político, económico y social.

\section{ADISCURSO HEGEMÓNICO: MODELOS DE INTERVENCIÓN Y AGENTES DE CAMBIO}

Volviendo al ejemplo inicial. En la práctica comunitaria que coordine en 2014 durante el primer semestre, concluimos un diagnóstico comunitario que hicimos en la Colonia Agrícola Oriental de la Ciudad de México y, después del periodo vacacional, retomamos la segunda parte de la práctica.

Cabe describir brevemente a la Colonia Agrícola Oriental, perteneciente a la Delegación Iztacalco de la Ciudad de México. La colonia abarca aproximadamente el $20 \%$ de la superficie de Iztacalco y es reconocida como una de las más grandes de la Ciudad de México (INEGI, 2002). Está dividida en tres cuadrantes: Agrícola Centro, Agrícola Oriental y Agrícola Poniente. La colonia se encuentra asentada en lo que fue el Lago de Texcoco y des $t$ tacó por ser un centro de producción agrícola con un peculiar sistema de chiาnampas, conocido como "[...] el más importante centro abastecedor de la ciudad de México y sus alrededores" (RIVERA, 
2002, p. 6). Sin embargo, debido a los proce 5 sos de industrialización y urbanización que influyeron en la ciudad, las activiาdades económicas se desplazaron hacía los sectores secundario y terciario. Por ello, ubicamos a la colonia en el sector urbano-popular de acuerdo con la clasificación de Stern (2010, p. 163) en tanto que, entre otros puntos: hay un alto porcentaje de concentración de la población, cuenta con servicios urba $n$ nos y sanitarios, las familias que la componen son clasificadas como nuclear y extendida, y en ella se desarrollan actividades culturales y recreativas. Los establecimientos comerciales, formales y principalmente informales, ocupan una buena parte de las avenidas y calles principales. La zona industrial, ubicada al oriente de la colonia, tiene fábricas como Cemex, El Globo, Coca-Cola, Reactivos y Limpieza Dogo. En esta zona, las viviendas están siendo removidas para construir edificios que alberguen a mayor cantidad de po-blación, situación que satura el uso del suelo -incluso notamos que estos ediาficios ahora tienen acabados más ostentosos para atraer a sectores de clase me $\mathrm{dia}$ - No obstante, la colonia se reconoce como de tipo popular y se caracterinza por problemas sociales como pobreza, desigualdad, desempleo e inseguridad. Estos y otros problemas se reflejaban en distintas notas periodísticas que revisamos, información que fuimos corroborando a lo largo del año 2014 mien-tras realizábamos la práctica comunitaria.

El diagnóstico arrojó como uno de los temas más importantes el embarazo adolescente, problemática reiterada tanto en medios de comunicación, como en las narrativas de las y los jóvenes de la colonia y personal de distintas instituciones en esta localidad ${ }^{3}$. En el grupo escolar surgieron diversas propuestas de intervención, entre ellas: pláticas, foros de discusión, campañas de difusión, cine debate, etc. Al momento de insistir en clarificar el objetivo de hacer alguna de estas actividades, las respuestas fueron: para ayudar

\footnotetext{
3 El tema del embarazo adolescente ha cobrado importancia en los últimos años en México. Incluso en 2015 el Gobierno de la República, creó la Estrategia Nacional de Prevención del Embarazo Adolescente. Si consideramos solo dos fuentes, se dice que la población entre 12 y 19 años que ha iniciado su vida sexual pasó de 15\% en 2005 al 23\% en 2012 según la Encuesta Nacional de Salud y Nutrición; otro dato es que el porcentaje de nacimientos en madres adolescentes aumentó entre los años 2003 y 2012, de $15.6 \%$ a 18.7\%, según el Gobierno de la República (PÉREZ et al., 2016).
} 
a los jóvenes a que reflexionen sobre su sexualidad, que conozcan los métodos anticonceptivos, evitar el embarazo adolescente, concientizar a los jóvenes, concientizar a las familias, siendo estas el principal núcleo de la sociedad, etc. Es decir, las propuestas se formulaban bajo un velo salvacionista, considerándolo como un efecto del fenómeno colonial, que, por un lado, cristaliza a los sujetos con ciertas características uniformes o esencialistas (en el caso de las madres y los padres adolescentes es que no son maduras/ os y les falta conciencia); también, ésta retórica nos posiciona como aquellas y aquellos profesionales que podemos rescatar a grupos en situación de vulnerabilidad.

Cabe mencionar que al momento de proponer algún contenido concreto en las actividades, solían ser muy similares a las propuestas de las instituciones que se localizan en la colonia y, que no siendo menos importante, el estudiantado criticó duramente mientras elaborábamos el diagnóstico. Fue evidente la reiteración discursiva y la gran similitud entre lo que postulaban las y los estudiantes y los mensajes institucionales. Esto llama la atención porque ¿cómo fue que los y las alumnas de quinto semestre aprehendieron de forma tan eficaz uno de los discursos disciplinares que circulan sobre trabajo social?

Los trabajadores sociales buscan el mejoramiento social, pero en su mayoría lo hacen para ayudar a las personas, las familias y pequeños grupos sociales como parte de su trabajo profesional [...] [El] trabajo social tiene por objeto adaptar el movimiento social y el cambio para que sea más manejable, ayudar más a los individuos, particularmente aquellos que son pobres y desfavorecidos (PAYNE, 2006, p. 1).

Trabajo social es una disciplina de las ciencias sociales que tiene por objeto de estudio la intervención social con sujetos concretos -individuales o colectivosque tienen un problema o una carencia social en un momento determinado. Su acción deviene de lo social y recae en lo social, precisamente en el punto de intersección que genera la relación sujeto, problema, contexto (TELLO PÉON, 2008, p. 1).

Este discurso es hegemónico, una de sus características es que reitera una identidad propia y en ocasiones inherente al trabajo 
social, por ejemplo, esto solo lo hacemos en trabajo social o ninguna profesión podría hacerlo, sólo trabajo social. Segundo, postula quesus bases son la justicia social y en otras ocasiones el empoderamiento de los actores/agentes sociales. Tercero, se manifiesta la profunda inquietud por dar respuesta a los problemas de los sujetos a través de intervenciones metodológicamente sustentadas. Siendo así, la intervención desde trabajo social se concibe como:

[...] un actuar 'dirigido' a dar respuesta a
determinados problemas y necesidades sociales
que presentan las personas, y para poder lograr el
objetivo esperado deberá tener en cuenta algunos
principios metodológicos que posibiliten crear las
condiciones necesarias para que se dé el cambio o,
en su caso, la estabilización del cambio conseguido
(HERNÁNDEZ ARISTU apud GALEANA DE LA; TELLO
PÉON, 2010, p. 27-28).
[...] una acción racional, intencional, fundada en
el conocimiento científico, que tiene por objetivo
desencadenar procesos de cambio social. La
participación del sujeto es indispensable, para hablar
de una acción de trabajo social [...] La intervención
marca un comienzo provocado, opera un cambio
en el curso de la situación problema mediante el
ejercicio de un saber que hace que suceda tal o cual
acontecimiento (TELLO PÉON, 2008, p. 1-2).

Desde esta perspectiva, el profesional en trabajo social es un agente de cambio o potenciador del mismo, es decir, durante su formación se insiste en su papel destacado al potenciar, movilizar, mostrar o desarrollar ciertos comportamientos, habilidades o posicionamientos en otros sujetos.

Ante esto, resalto dos cuestiones que me parecen temas a profundizar. Un primer elemento, es que la intervención se concibe como un campo bipolar, de unos hacia otros, donde el contexto figura como telón de fondo pero no como elemento que nos constituye como sujetos sociales. Así, se establece que la intervención se puede lograr si se siguen una serie de pasos previamente comprobados en la práctica; suele estipularse que la intervención es necesaria para el cambio, como una vía civilizatoria y unidireccional hacia el bienestar, englobado como 
cambio social. Y una segunda cuestión es que desde algunas miradas, no se problematiza a quien ejerce o ejercerá el trabajo social, los problematizados son los otros, los de afuera.

Hay una cuestión que me parece importante para aterrizar los escritos revisados en torno a esto (PAYNE, 2006; TELLO, 2008; HERNÁNDEZ ARISTU, 2010), y es el momento en que el discurso se consolidó con este nuevo lenguaje: modelos de intervención, actores y agentes de cambio, desarrollo humano, etc. Quiero decir, algunos de estos conceptos pudieran tener su origen con mucha antelación a la época actual pero ¿en qué momento histórico se conformó y confirmó este lenguaje común en el trabajo social, es decir, existió consenso y por lo tanto se consolidó? ¿Por qué si no hablamos en estos términos perdemos interlocución, no sólo con las instituciones, sino entre colegas, y las/los estudiantes se sienten desorientados preguntando con incertidumbre, entonces qué es trabajo social? Sin tener una respuesta definitiva, puedo afirmar que este nuevo lenguaje dio señales en la década de los años 90, un momento histórico por demás abordado por historiadores, antropólogos y sociólogos: "Está de moda decir que en 1989 el año de la caída del socialismo marcó el fin de las utopías. Y yo en cambio creo lo contrario, que en realidad marcó la última década de explosión de una utopía que en este caso fue la utopía liberal capitalista" (ŽıŽEK, 2003, p. 5). Este lenguaje es producto de esa utopía en la fase neoliberal.

Por neoliberalismo, concebimos dos principales cuestiones. Por un lado, como una fuerza estructural que afecta las oportunidades de vida de las personas y, por otro lado, como una ideología de gobernanza que configura subjetividades (GANTI, 2014, p. 89). Uno de los sustentos más importantes del neoliberalismo es el liberalismo clásico, que se basa en la creencia de que el bien social no es natural sino que deviene de la concertación del esfuerzo político y principalmente la organización. Por esta razón, en la fase neoliberal, se ha establecido un marco legal e institucional que promueve una actividad gubernamental predecible bajo un orden competitivo que deberá ser efectivo, donde el mercado se exalta y, que en comparación con el Estado, parece un mecanismo más eficiente. Esto no significa que se eliminará el Estado, sino que se buscará redefinir su naturaleza y sus funciones (GANTI, 
2014, p. 92). Es importante resaltar que las políticas neoliberales estarán mediadas por género, clase, raza, edad y otras categorías que apuntan a las diferencias sociales (GANTI, 2014, p. 95) y que quienes desplegamos estrategias de intervención no podemos obviar, es decir, nuestras propuestas dependerán de nuestra ubicación en la cartografía más amplia y nuestra ubicación en la red de estas categorías.

Las peculiaridades de la sociedad capitalista contemporánea no pueden ser tomadas únicamente como telón de fondo, éstas son las que cotidianamente nos atraviesan a los sujetos, de ahí el interés por profundizar en el estudio de la subjetividad para debatir en torno a la intervención en trabajo social. Entre otras cosas, para tratar de entender cómo se encarnan en los sujetos, los intrincados procesos estructurales diferenciadores. Así, la práctica profesional no está exenta -de hecho nada más alejado de ello- del impacto del paradigma de la democracia, de la ideología neoliberal ${ }^{4}$, la sociedad de consumo (a la que no acceden la mayoría de las personas que viven en pobreza y pobreza extrema5), es decir, las prácticas profesionales debe analizarse a la luz de las condiciones materiales que traza el capitalismo neoliberal ${ }^{6}$.

Por ejemplo, la incertidumbre crónica por la volatilidad extrema de la economía juega un papel fundamental en las elecciones que toman las personas, así, la agencia se ejerce pero no libremente, está determinada por la estructura que no es libre ni racional. "Agencia, elección, oportunidad, como experiencias, ahora necesitan ser entendidas como adherencias en historias particulares que se producen materialmente, socialmente $y$

\footnotetext{
4 “Una ideología [... ] no es necesariamente 'falsa': en cuanto a su contenido positivo, puede ser 'cierta', bastante precisa, puesto que lo que realmente importa no es el contenido afirmado como tal, sino el modo como este contenido se relaciona con la posición subjetiva supuesta por su propio proceso de enunciación [...] Hoy, en el capitalismo tardío, cuando la expansión de los nuevos medios masivos [...] permite que la ideología penetre eficazmente en cada poro del cuerpo social, el peso de la ideología como tal ha disminuido" (ŽIŽEK, 2008, p. 14-23).

${ }^{5} \mathrm{Y}$ aunque existen múltiples programas sociales, estos no inciden directamente en aminorar, o mejor aún, eliminar este porcentaje.

6 Retomando la clasificación que establece Pansza (2005) de prácticas profesionales dominantes, emergentes y decadentes.
} 
culturalmente" (NAROTZKY; SMITH, 2006, p.90). Es decir, las oportunidades que se generan para las personas están determinadas por los rumbos que toma la producción capitalista y si bien en el acercamiento a los grupos podemos dar cuenta de que existe albedrío, éste debe ser sopesado con relación a los sujetos y muchas otras variables. En este sentido, “[... ] las narrativas políticas fragmentadas y la profunda ambivalencia hacía la agencia ejercida en la arena pública política, [ocurre gracias a las] tácticas diarias y privadas de acción evasiva" (NAROTZKY; SMITH, 2006, p. 193), que presionan a los sujetos.

Este nuevo lenguaje que alude al fortalecimiento del capital social, reitera que aquello que puede ser catalogado como exitoso son quienes pueden generar movilidad dentro del capital. Desde esta postura las intervenciones exitosas son aquellas que logran cambios sin trastocar la estructura, y esto, se da por sentado que no ocurrirá. En ese sentido, el capital social invisibiliza el sistema de explotación bajo el cual está sustentado y en muchos casos, es velado cuando hablamos de exclusión porque así despolitizamos la explotación. Al mismo tiempo, hay un repliegue de la confrontación, se considera que no puede haber conflictos y se obliga a pensar en compromisos y responsabilidades que, por supuesto, recaen con mayor frecuencia en los subalternos, por lo cual, recae una doble o triple sujeción para las personas con las que trabajamos, a través de valores creados cultural y selectivamente sobre los ellos.

\section{PRACTICANDO LA TEORÍA: ATENDER A LAS CONTRADICCIONES}

Según sus detractores, el marxismo es un tipo de teoría necesariamente reductiva y determinista: no se permite a ninguna actividad que sea real y significativa por sí misma, sino que es siempre reducida a una expresión directa o indirecta de algún contenido económico precedente [... En realidad es

formulada a menudo con una confianza tan firme como anticuada. Sin embargo, difícilmente puede negarse que proviene, con todas sus dificultades, de una forma corriente de marxismo (WILLIAMS, 1980).

Raymond Williams, es considerado un estudioso de la teoría cultural marxista, su aportación sobresalió porque trabajó una revisión del marxismo no desde una visión economicista y 
“objetiva”, por el contrario, advirtió los aportes del marxismo en el estudio de la subjetividad.

\begin{abstract}
Williams siempre escribe de un modo situado y esto significa, antes que nada, pensarse desde un lugar. Pensarse desde un lugar es tomar una distancia [... Este lugar desde el que se piensa es así, por un lado, el propio emplazamiento persona, que nos localiza en lo íntimo; pero también, en la medida en que es relativo, es la distancia crítica que se toma con respecto a estas posiciones, que las relativiza y las inserta en la historia colectiva (GARCÍA RUÍz, 2008, p. 14).
\end{abstract}

Su libro "Marxismo y Literatura" (WILLIAMS, 1977) resulta interesante en varios sentidos. Trabaja a detalle conceptos nodales en Marx - considera que hay una distancia entre Marx y el marxismo - , tomando como puntos de referencia algunas críticas hechas por los opositores de esta línea de pensamiento, pero su objetivo va más allá y es cuestionar a los propios marxistas en las elaboraciones simplistas y lecturas abstractas.

Por ejemplo, respecto a la proposición de que en el capitalismo existe una base determinante y una superestructura, donde comúnmente se afirma que la primera es el determinante económico y la segunda lo ideológico, Williams revisa las distintas definiciones explícitas e implícitas de ambas proposiciones, incluidas las que están en los textos de Marx, y afirma que es posible considerar tres sentidos de la superestructura: a) Las formas legales y políticas que expresan relaciones de producción existentes; b) las formas de conciencia que expresan una particular concepción clasista del mundo; y, c) un proceso en el cual, a través de toda una serie de actividades, los sujetos tomen conciencia de un conflicto económico fundamental y lo combatan. Es decir, desmenuza todos los sentidos (dominantes y emergentes) del término y señala que siendo así, superestructura no es sinónimo de ideología como forma abstracta, sino que apunta hacia las interrelaciones entre a) las instituciones; b) las formas de conciencia; y c) las prácticas políticas y culturales (WILLIAMS, 1980).

Con ello, demuestra que Marx no pensaba en la separación de áreas de pensamiento y actividad como en la separación de conciencia (lo ideológico) y la producción material (lo económico). 
[... ] cuando Marx llegaba a cualquier tipo de análisis probado o tomaba conciencia de la necesidad de un análisis de este tipo, se manifestaba a la vez específico y flexible en la utilización de sus propios términos $[\ldots]$ En todo análisis marxista serio las categorías no son utilizadas de modo abstracto (WILLIAMS, 1980, p. 11).

Lo que me parece relevante de este análisis, es que Williams trabaja teóricamente los conceptos de Marx, a la luz de las críticas, de los argumentos de otros marxistas y de su comprensión de la sociedad capitalista, no como un todo homogéneo y generalizado, de ahí su interés por el estudio de la cultura hegemónica y sus rupturas. Ello le permite afirmar que dentro del proceso social total, las determinaciones pueden ser experimentadas individualmente pero son siempre actos sociales que mantienen relaciones muy complejas.

Por lo tanto, la 'sociedad' nunca es solamente una 'cáscara muerta' que limita la realización social e individual. Es siempre un proceso constitutivo con presiones [...] que se expresan en las formaciones culturales, económicas y políticas y que, para asumir la verdadera dimensión de lo 'constitutivo', son internalizadas y convertidas en 'voluntades individuales'. La determinación de este tipo [...] se halla en el propio proceso social en su totalidad, y en ningún otro sitio; no es un abstracto 'modo de producción' ni en una 'psicología abstracta' (WILLIAMS, 1980, p. 15).

¿Cómo pensar a la profesión desde este marco? Primero, es considerar que las formas de conciencia que atraviesan a una disciplina están enmarcadas en una concepción peculiar del mundo, por tanto, impactaran las actividades, las concepciones sobre el Otro, los sujetos y delineará los caminos a seguir. En el ejemplo del grupo escolar que expuse anteriormente, se cristalizó en la forma como los jóvenes formándose como trabajadores sociales representaban a jóvenes trabajadores de la Col. Agrícola Oriental, la tarea del estudiante era concientizar a los jóvenes para que usen métodos anticonceptivos y se redujera el embarazo a temprana edad. Así los caminos a seguir que se planteaban iban en esa dirección y era impensable brindar como opción la interrupción legal del embarazo, por ejemplo. En ese sentido, fue importante 
dar un paso atrás como grupo, decir no hay urgencia de intervenir sino de discutir ¿por qué esas son nuestras opciones y no otras? ¿Cómo imagino y pienso a los jóvenes trabajadores y por qué? Poco a poco comenzamos a hablar, por ejemplo, del reconocimiento de la vida sexual temprana y su nula relación con la reproducción, además de que para su ejercicio pleno es fundamental que existan condiciones materiales que les permitan a los jóvenes tener vida sexual sin esconderse, ni ejerciéndola en lugares públicos que les ponen en riesgo o concebir (por estas presiones y límites) que si tienen sexo deberán asumir la responsabilidad con la maternidad y paternidad obligatoria.

Por otro lado, tal como se ha enmarcado la intervención profesional en trabajo social, no tiene que ver únicamente con los debates disciplinares sino con la interrelación entre las instituciones, las políticas y las prácticas culturales, es decir, debe buscarse entendimiento dentro de un proceso social total, porque aunque experimentemos esos malestares como únicos de la profesión, no nos competen únicamente a nosotras/os. Finalmente, considero que las reflexiones de Williams (1980) bien pueden ayudarnos para develar que las dicotomías que atraviesan nuestros discursos disciplinares no corresponden con el proceso social total del que derivan las problemáticas sobre las que trabajamos cotidianamente, de ahí que nos limiten en su comprensión, por tanto, en la intervención.

Marx nos advirtió [que] [p]ara poder actuar coherentemente en el mundo tenemos que indagar bajo las apariencias superficiales, ya que estas suelen inducir a una actuación con resultados desastrosos [...] reconocer la posibilidad general de que a menudo atendamos a los síntomas más que a las causas subyacentes y de tener que desenmascarar [la contradicción,] lo que sucede verdaderamente bajó múltiples capas de apariencias superficiales a menudo engañosas (HARVEY, 2014, p. 20).

La vigencia de los descubrimientos de Marx, reside en que podemos retomar sus argumentos para contestar fenómenos que observamos en la actualidad, por ejemplo: contestar la premisa de que el sistema de propiedad privada junto con la igualdad de derechos garantiza un crecimiento económico a largo 
plazo; que el sistema capitalista si es eficiente económicamente se traducirá a los sujetos, esta premisa no se sostiene porque existe un antagonismo entre las clases sociales con intereses, por definición, irreconciliables; y, que la igualdad jurídica y la libertad, asentada en papel y que proclamaba (y aún lo hace) el liberalismo suelen ser maniobras que no se cumplen para la mayoría de la población (SEGUí, 2010). En el mismo sentido, Marx subrayó la importancia de indagar bajo las apariencias superficiales para desentrañar aquello que está latente pero quizá oculto (como el trabajo dentro de la mercancía) y mostró el carácter sontológicamente injusto〉 del capitalismo. Traído a nuestro tema, vale más discutir las políticas o la intervención mismas que sólo cómo se aplicaran porque "[...] políticas erróneas basadas en una teorización errónea agravan las dificultades económicas y exacerban los trastornos sociales y la miseria resultante" (HARVEY, 2014, p. 25).

\section{POSIBLES HORIZONTES EN EL TRABAJO SOCIAL MEXICANO}

[Roseberry] Buscó afanosamente narrativas éticas, no en un sentido de absolutos sino en el sentido de que debían ser adecuadas frente a la complejidad y las profundidades de la experiencia humana. Fue capaz de encarar la incertidumbre y la vigilancia intelectual (REBEL, 2004).

Con el grupo de práctica comunitaria se generó una discusión interesante a partir de los textos sobre intervención desde distintas disciplinas. El alumnado externó críticas a las diferentes propuestas, por ejemplo: algunos son modelos esquemáticos; no consideran "lo social", se concentran en el individuo; no exponen las dificultades inherentes de la intervención, etc. También comentaron que cuando ellas y ellos han sido participes como sujetos a quienes se dirigía una intervención, en ocasiones, han identificado el desinterés real de estos profesionales, se enfrentan a trámites burocráticos que les hace sentir como cifras y no como sujetos ${ }^{7}$. Una de las propuestas que les pareció sugerente ${ }^{8}$ implicaba

\footnotetext{
7 Esta reflexión se dio con la lectura Modelos de orientación e intervención psicopedagógica. Modelos de intervención por servicios (2004) de Esperanza Bausela Herrera.

8 "Trabajo comunitario desde una perspectiva psicoanalítica. Un acompañamiento
} 
un trabajo sin límite de tiempo y eso generó interrogantes sobre ¿qué tan válido es asumir un trabajo así, a largo plazo y dejando en todos los implicados la decisión de cuándo cerrar el proceso? Otra de las lecturas les hizo pensar en la intervención no como un fin sino como estrategia ${ }^{9}$ y reflexionaron sobre las posibilidades ilimitadas de eso que analíticamente denominamos "lo social", lo que no se puede calcular. Comprendimos en conjunto que la problemática de una colonia como la Agrícola Oriental, sólo la podemos entender si profundizamos en las interrelaciones (visibles o no) que tiene esta localidad con el contexto regional y global. Ellas y ellos -el estudiantado en ese momento y ahora como profesionales - se asumieron también como sujetos de intervención ¿qué de sus experiencias y saberes necesitan poner en el análisis y las propuestas, para un trabajo concreto como era esta práctica comunitaria?

Como parte de este proceso, puse a discusión que debemos considerar la investigación, para este caso el diagnóstico comunitario, ya como una intervención. Estamos en campo, somos ajenos, aun cuando vivamos ahí y ellas/ellos eran jóvenes como los sujetos con quienes conversaban, nuestra posición nos sitúa en otro lugar, con una distancia que nos permite desnaturalizar aquello que la cotidianidad encubre. Decir que la intervención es la práctica y la revisión de literatura o el diagnóstico es la teoría, reafirma la separación entre teoría y práctica. Ello ha contribuido, entre otras cosas, a considerar trabajo social como una profesión apta para la práctica pero no para hacer ni profundizar en la teoría, dejando la investigación como especialidad de otras profesiones. Profundizar en el estudio y análisis de lo social como un todo económico, político y cultural, nos permite posicionarnos en cualquier área o línea de investigación ${ }^{10}$, además que vigilamos nuestra tarea intelectual, para así arribar a éticas narrativas que nos lleven más allá de compartir el malestar con un sector o indignación por una situación de vulnerabilidad, porque indagar sobre las rupturas de lo hegemónico no es tarea rápida y evidente,

en la construcción grupal de saberes (2011) de Verónica Egas y Emilio Salao.

9 Intervenciones en el espacio público: performance, mirada y ciudad (2013) de María Laura González.

${ }^{10}$ Sin ceñirnos a los programas sociales y ampliando nuestra injerencia profesional. 
$[\ldots]$ por ejemplo, $[\ldots]$ un 'Estado', $[\ldots]$ nunca para de hablar[pero] no tiene público; o más bien, tiene varios públicos que escuchan cosas diferentes; y quienes, al repetir lo que el Estado dice a otros públicos, cambian las palabras, los tonos, las inflexiones y los significados (ROSEBERRY apud REBEL, 2004, p. 187).

Es decir, el Estado no puede concebirse homogéneamente, mucho menos lo que entendemos por sociedad civil desde una visión liberal, siendo así, nuestros objetos y sujetos de intervención no están dados, no pueden ser evidentes porque se construyen y esto requiere pensar, reflexionar y analizar, tomar un tiempo para ello. No existe algo como un catalogo teórico para ver cuál teoría se acopla mejor a lo que percibimos en la vida cotidiana, suele ser más complejo que eso, porque me juego yo también. Con ello quise insistir que la intervención no es unidireccional, sino para que sea efectiva debe pensarse dialécticamente y como un proceso que me trastocará. Yo no puedo ser la misma persona a lo largo y después de esa intervención, además de que implica que yo deba ubicarme y desmitificar mis propias representaciones del mundo. Regresando al ejemplo de embarazo adolescente, formulaba lo siguiente: el cambio social que intrínsecamente sugiere un cambio lineal, no está dado por sentado ¿qué pasa, si la intervención que despleguemos incita a las y los jóvenes a tener más de un hijo en un periodo corto de tiempo? Con ello quería evidenciar que cuando hablamos de cambio social sin definir explícitamente qué entendemos por cambio, ni definimos el horizonte ideológico y material que sustentará dicho cambio, podemos caer en visiones reduccionistas, porque damos por sentado que los sujetos deben ir en una dirección, esa que yo tengo en mente como la ideal.

Así que preguntaba ¿qué sitio ocupo en la reiteración de la práctica dominante o emergente de la profesión? Y por supuesto, tiene que ver en cómo me posiciono frente a las políticas y el contexto que me atraviesa, porque de lo contrario se corre "[... ] el peligro de la formalización de las teorías sociales: reducirlas a enfoques clasificatorios, mecanicistas, vaciándolas de su naturaleza y de su potencial explicativo, actitud que refuerza el empirismo en la práctica profesional" (IAMAMOTO, 2000, p. 94). Y esta es una preocupación mayor, la de no concebir a la teoría como "[... ] teoría 
de las posibilidades de acción" (IAMAMOTO, 2000, p. 101), sino solo como marco explicativo pero que no trastoca la realidad empírica.

Lo que es patente en nuestra formación profesional como trabajadoras/es sociales en México, es un formalismo teórico que tiene impacto metodológico, este "[...] formalismo se operacionaliza en la práctica, principalmente a través del congelamiento del método en sucesivas démarches de conocimiento/ diagnóstico/ planificación/ ejecución /evaluación" (DE PAULA FALEIROS, 2000, p. 106). Conocido como el método básico, es el más connotado en la enseñanza superior y es en la formación de posgrado donde se pone en cuestión y se reflexiona sobre otros procesos. El método básico, a su vez, tiene impacto en la mecanización teórica, porque al enfatizar su aplicación, acotamos la importancia de la teórica. Por tanto, "[...] [e]s preciso la construcción de mediaciones teóricas y críticas de esas clasificaciones inmediatas [...]" (DE PAULA FALEIROS, 2000, p. 111) y superficiales con las que determinamos nuestro entorno, su problematización y, en breve, su resolución.

El desarrollo de [la] acción no es separado del conocimiento. Al contrario, sin el conocimiento sólo queda el activismo que significa práctica mimética e inconsecuente. Sólo la construcción del análisis puede superar el mimetismo y el activismo, y reflexionar sobre las consecuencias de las prácticas. El conocimiento es la condición del fundamento de la acción (DE PAULA FALEIROS, 2000, p. 113).

Debemos asumir que los discursos se materializan y tal como se entiende la intervención, es más cercana a la idea imperante de democracia liberal en el capitalismo, que a una forma contestataria y de ruptura con las normativas del libre mercado. Un consenso hegemónico existe como una frágil alianza conceptual-política que sirve forzadamente en todo momento a dominar los discursos de los que están sujetos mediante formaciones de lenguaje que normalizan la opresión, pero también sirve para reacomodarse a sí misma como una alianza ficticiamente coherente (REBEL, 2004, p. 185). Por ello,

[...] las obras contrahegemónicas son inventos narrativos [...] liberados de las lógicas ordenadas (es decir, naturalizadas) de identidad y autenticidad 
y capaces, aunque no siempre con esta intención, de perturbar hasta el punto de la incapacidad, las habilidades específicas de las negociacionesnarrativas de mantener íntegro el bloque hegemónico (REBEL, 2004, p. 183).

En este sentido, una ruptura posible es plantear la praxis del trabajo social. Por praxis entendemos una práctica que abre condiciones de posibilidad, movilización y acción estratégica, frente a los programas políticos con los que nos enfrentemos en nuestra práctica profesional en el espacio social, porque si bien sabemos que hay elementos que facilitan la reproducción de condiciones también es cierto que a lo largo del tiempo se producen contrafuerzas (SMITH, 2014, p. 19-20). Por ello, señalaremos cuatro puntos que son aportes para acercarnos a la praxis como trabajadoras y trabajadores sociales.

Primero, es atender a las tensiones que observamos entre lo instituido que está establecido como norma; lo instituyente, que está latente a nivel de la práctica cotidiana; y, las contradicciones que observamos entre las políticas institucionales y las condiciones materiales de existencia de los grupos y sectores a quiénes están dirigidas. Hacerlo implica ver esas contradicciones como síntomas y contextualizar ese segmento de realidad que nos interesa abordar. Dejar de culpar o enfatizar que el problema reside en los sujetos con los que trabajamos

No debemos olvidar el contexto más amplio y enlazarle siempre con el actual régimen de trabajo, “[...] las transformaciones sociales contemporáneas, operadas por la programática neoliberal bajo el comando del capital financiero trae nuevos desafíos [... ] al conjunto de hombres y mujeres que viven de la venta de su fuerza de trabajo" (MONTAÑO, 2007, p. 1). Esto nos permite no dejarnos arrastrar por la indiferenciada diversidad y correr el peligro de aquilatar por igual todo aquello que encontremos en nuestro espacio profesional. No significa tomar sólo una categoría analítica, habrá que tomar las que sean necesarias para comprender desde un sentido amplio lo que nos interesa abordar, pero sin olvidar el eje explicativo para ese fenómeno específico y muchos otros. En esa medida, encontraremos fuerzas, relaciones y experiencias que son determinantes de la subjetividad social. 
Tercero, el análisis contrapunteado, me refiero entre teoría y experiencia empírica/práctica o tomando distintos puntos de análisis sobre un tema, debe prevenirnos de trabajar bajo el velo salvacionista, posibilitando una amplia comprensión del terreno que pisamos. A partir de abstracciones, posicionar una mirada dialéctica que permita trascender las apariencias sin colocarnos como sujetos salvadores. El análisis contrapunteado, nos permite ubicarnos como parte del problema, ver a los sujetos en sus múltiples facetas y privilegiar el análisis antes que intervenir para develar qué hay bajo las apariencias superficiales de una problemática.

Cuarto, rastrear un análisis sobre las políticas del Estado, no entendiendo a este último como entidad unificada, cabe pensar al Estado como ente dislocado, como una variedad de modalidades y técnicas hacia los grupos sociales que nos obliga a mirar las prácticas rutinarias y los diferentes niveles de ese Estado. Ello nos permitiría contar con un marco analítico sobre las aproximaciones funcionales e institucionales del Estado, que articulan construcciones ideológicas y culturales, así como sus representaciones; para comprender sus conexiones constitutivas entre la economía política, la estructura social, el diseño institucional, la práctica diaria y su representación (GUPTA, 2012). En trabajo social, a través de nuestra práctica profesional estamos cerca de los distintos niveles, áreas o departamentos que conforman el Estado. Ello no significa, en automático, que podemos trasladar esa observación para definir al conjunto, pero sí permite contar con un punto de análisis y construir un conocimiento situado acerca de ese espacio estatal. Esta ubicación analítica, nos permitirá aprovechar las contradicciones y tensiones entre múltiples agencias, departamentos y organizaciones, para rastrear sus prácticas de cuidado y protección, y aquellas que son violentas y de exclusión bajo el velo de la normalización.

\section{CONSIDERACIONES FINALES}

En este ensayo discutimos algunas premisas que se reiteran constantemente en el discurso hegemónico del trabajo social mexicano. La problemática está situada en la dicotomía investigación e intervención, específicamente en lo que entendemos por teoría y práctica. Para el desarrollo de ideas, 
retome mi experiencia como profesora en la Escuela Nacional de Trabajo Social en la UNAM y el trabajo de Raymond Williams (1980). Éste último, es pertinente porque él apunta que todo análisis teórico debe anclarse en el proceso social en su totalidad, cabe decir, asumiendo que esta tarea será inacabada. No hay análisis serio sino advertimos que el proceso social en su totalidad sólo se puede dividir analíticamente, debemos cuestionamos por qué excluimos o nos excluimos del análisis. Si bien, la práctica no es privativa de tres años de la carrera (para el caso de México), tampoco la teoría es tema de sólo cinco semestres. Toda dicotomía es sospechosa porque nada puede ser reducido a un campo bipolar, siendo ésta la característica de las reducciones que observamos en base-superestructura, individuo-sociedad, público-privado, femenino-masculino y teoría-práctica.

Afirmar que todo nuestro quehacer se centra en la intervención, provoca que el alumnado la demande y nos impide valorar en amplio sentido el pensamiento y la reflexión, momentos necesario para comprender algo en su complejidad, para visibilizar los lazos de su especificidad con lo estructural y para apostarnos en un proyecto que vaya más allá de ser alternativo. Porque la práctica social desde trabajo social no se revelará por el simple hecho de estar en campo. Ante la pregunta ¿es la intervención el acto distintivo de trabajo social? Respondemos que no, sustentado en la revisión de las intervenciones que se hacen desde otras disciplinas. No obstante, sí puede haber una praxis desde trabajo social y señalamos cuatro aspectos: atender a las contradicciones sociales, hacer el esfuerzo por ubicarnos y ubicar las problemáticas en el contexto capitalista, apostar por un análisis contrapunteado para evitar la retórica salvacionista y rastrear las políticas del Estado sin concebirlo como una entidad unificada.

Laexperiencia conelgrupodepráctica comunitariafue determinante para las personas que estuvimos involucradas y que tuvo como resultado apostar, para ese momento, más a la discusión que a una intervención inmediatista. El resultado de estas discusiones derivó en un artículo que fue nuestra primera experiencia en ese sentido. Para mí, en coordinar un ejercicio colectivo de escritura y para ellas y ellos tener su primer artículo publicado. 


\section{REFERÊNCIAS}

BAUSELA HERRERA, E. Modelos orientación e intervención psicopedagógica. Modelos de intervención por servicios. Revista Iberoamericana de Educación, 2004. Disponível em: <http://www. rieoei.org/deloslectores/823Bausela.PDF>. Acesso em: 4 mayo 2017.

DE PAULA FALEIROS, V. La cuestión de la metodología en Servicio Social: reproducirse y representarse. En: Borgianni, E. y C. Montaño (Orgs.). Metodología y Servicio Social: hoy en debate. São Paulo: Cortez, 2000. p. 104-120.

EGAS, V.; E. SALAO. Trabajo comunitario desde una perspectiva psicoanalítica. Un acompañamiento en la construcción grupal de saberes. Revista Latinoamericana de Ciencias Sociales, v. 2, n. 9, p. 899-911, 2011. Niñez y Juventud.

GALEANA DE LA O, S.; TELLO PEÓN, N. Fragilidad y debilidad del discurso en trabajo social: ausencia de la construcción desde la intervención social. Revista Trabajo Social UNAM, Ciudad de México, VI Época, n. 1, p. 22-35, dic.2010.

GANTI, T. Neoliberalism. Annual Review of Anthropology, Palo Alto, n. 43, p. 89-104, 2014.

GARCÍA RUÍZ, A (Edición). Raymond Williams. Historia y cultura común. Madrid: Los libros de la Catarata, 2008.

GONZÁLEZ, M. L. Intervenciones en el espacio público: performance, mirada y ciudad. Revista Brasileira de Estudos da Presença, Porto Alegre, v. 3, n. 3, p. 727-741, 2013.

GUPTA, A. Red tape: bureaucracy, structural violence, and poverty in India. United States of America: Duke University Press, 2012.

HARVEY, D. Diecisiete contradicciones y el fin del capitalismo. Quito: Instituto de Altos Estudios Nacionales del Ecuador (IAEN), 2014.

HERNÁNDEZ ARISTU, J. Participación ciudadana y mediación social. Una reflexión desde la práctica del asesoramiento social. Mediaciones Socials, Madrid, n. 7, p. 113-142, 2010.

IAMAMOTO, M. La metodología en el Servicio Social: lineamientos para el debate. En: Borgianni, E. y C. Montaño (Orgs.). Metodología y Servicio Social: hoy en debate. São Paulo: Cortez, 2000. p. 93-104. 
INEGI, Instituto Nacional de Estadística y Geografía MÉXICO. Sistema para la Consulta del Cuaderno Estadístico Delegacional de Iztacalco. Distrito Federal, Edición 2002, en Cuadernos estadísticos municipales y Delegacionales. Disponível em: http:// www.inegi.gob.mx/.../cemo2/estatal/df/moo6/index.htM. Acesso em: el 10 de mayo de 2017.

MONTAÑO, C. Trabajo social e intervención: la politización de la acción profesional. En: COLOQUIO INTERNACIONAL DE ESTUDIANTES DE TRABAJO SOCIAL “DESARROLLO, POLÍTICA SOCIAL E INTERVENCIÓN PROFESIONAL”, 7.; 2007. Anais... Puno: Universidad del Altiplano, 2007.

NAROTZKY, S.; G. SMITH. Immediate struggles: people, power, and place in rural Spain. London: University of California Press, 2006.

PANSZA, M. Pedagogía y currículo. México: Gernika, 2005.

PAYNE, M. What is professional social work?. Bristol: Policy Press, 2006.

PÉREZ, B. et al. ¿Qué significa ser madre y padre adolescente? Estudio exploratorio del embarazo adolescente en un contexto urbano popular. In: Evangelista, A. et al. Género y Juventudes. México: El Colegio de la Frontera Sur, 2016. p. 229-258.

RAYMOND, W. Marxismo y literatura. Barcelona: Península, 1980. RAYMOND, W. Marxism and literature. New York: Oxford University Press, 1977.

REBEL, $H$. Después del siguiente genocidio: el modernismo reaccionario y el desafío postmoderno a la ética analítica. Recordando a Bill Roseberry. Arizona: Universidad de Arizona, 2004.

RIVERA, N. En la casa de la sal: monografía, crónicas y leyendas de Iztacalco. México (DF): Versal, 2002.

SEGUí, L. Vigencia de Marx (y del marxismo). En: Seguí, Luis (Comp.). Triunfo y fracaso del capitalismo: política y psicoanálisis. Malaga: Miguel Gómez, 2010. p. 17 - 29.

SMITH, G. Intellectuals and (counter-) politics: essays in historial realism. New York: Berghahn, 2014. 
STERN, C. El problema del embarazo en la adolescencia: contribuciones a un debate. México (DF): El Colegio de México, 2010.

TELLO PEÓN, N. Trabajo social, disciplina del conocimiento: apuntes de trabajo social. México (DF): Estudios de Opinión y Participación Social, 2008.

ŽıŽEK, S. El espectro de la ideología. In: ŽıŽEK, S. (Comp.).

Ideología: un mapa de la cuestión. Argentina: FCE, 2008. p. 7-42.

ŽıŽEK, S. La estructura de dominación y los límites de la democracia. In: CONFERENCIA IMPARTIDA EN LA FACULTAD DE FILOSOFÍA Y LETRAS, 2003. Buenos Aires: Universidad Nacional de Buenos Aires: 2003. Disponível em: <http://www.lacan.com/zizekbuenosaires.htm>. Acesso em: 4 mayo 2017. 